\title{
Spatio-temporal expression and stress responses of DGAT1, DGAT2 and PDAT responsible for TAG biosynthesis in Camelina sativa
}

\author{
Lixia Yuan',2, Xue Mao', Kui Zhao', Yan Sun', Chunli Ji', Jinai Xue', Runzhi Li" ${ }^{1 *}$ \\ ${ }^{1}$ Institute of Molecular Agriculture and Bioenergy, Shanxi Agricultural University, Taigu 030801, Shanxi, China, ${ }^{2}$ College of Biological Science \\ and Technology, Jinzhong University, Jinzhong 030600, Shanxi, China
}

\section{A B S T R A C T}

\begin{abstract}
Camelina sativa is embraced as a dedicated Brassicaceae oilseed for its valued seed oil wildly used for food, feed and biofuel, with commercial cultivation to increase largely across the world recently. This study was conducted to investigate the molecular mechanism underlying camelina oil biosynthesis and great environmental adaptability by focusing on phospholipid diacylglycerol acyltransferase $(P D A T)$, diacylglycerol acyltransferase type 1 (DGAT1) and type 2 (DGAT2) families. Following genome-based identification for the three family members, qPCR and GC analysis were employed, respectively, to examine expression patterns of these family members and lipid profiles in camelina seed and other tissues, particularly under stress conditions. It revealed that each of these families had three members in camelina. Those members showed various expression patterns in camelina seed development and other tissues. The tremendous expression of CsDGAT1-A and CsDGAT2-C appeared in seeds between 15 and $22 \mathrm{DAF}$, the period for vast oil enhancement, suggesting that CsDGAT1-A and CsDGAT2-C contribute to oil accumulation in seeds with CsDGAT1-A much important. CsPDAT3 and CsDGAT1-C highly expressed in leaf and flower, indicating that both enzymes, particular CsPDAT3 is the major contributor for oil accumulation in leaf and flower. Under cold stress, with oil elevating, expressions of CsDGAT2-B and CSPDAT1 were induced to increase in the seedlings by 11- and 6-folds respectively compared to the control, showing that CsDGAT2-B and CsPDAT1 mainly function for oil accumulation in plants beneficial for cold tolerance. Furthermore, salt stress induced CsDGAT1-B and CsPDAT2 to express up by 8-15 folds, demonstrating that CsDGAT1-B and CsPDAT2 play important roles in salt resistance. Collectively, different members of CsDGAT1, CsDGAT2 and CsPDAT work at different tissues and stress conditions, providing a new insight into the regulatory mechanism of oil accumulation, especially functions of CsDGAT1, CsDGAT2 and CsPDAT family members in plant stress responses.
\end{abstract}

Keywords: Camelina sativa; Oil and fatty acid biosynthesis; Diacylglycerol acyltransferase (DGAT1 and DGAT2); Phospholipid diacylglycerol acyltransferase (PDAT); Stress response

\section{INTRODUCTION}

Camelina (Camelina sativa L.) has been recognized as an important new oilseed for both food and nonfood purposes including biofuel and oleochemicals (Zanetti et al., 2013). This oil crop of Cruciferae family was cultivated in Europe, Central Asia and North America for about 3,500 years as a valued vegetable oil source. Currently, camelina is getting the rising interest and increasing expanding of cultivation across the world (Gugel et al., 2006; Mcvay et al., 2011; Guy et al., 2014).

With several agronomic advantages, camelina could be easily developed for commercial production of vegetable oil as much healthy food and a renewable resource for green manufacture of high-quality biofuels (Lu et al., 2008). Camelina oil contains $35-40 \%$ of linolenic acid (18:3n-3), an ideal source for $\omega-3$ fatty acid dietary supplement and the nutraceutical application (Gebauer et al., 2006). In addition, the oil contains lower level of erucic acid (22:1) compared to other brassica oil, a fatty acid related with cardiovascular disease (Iskandarov et al., 2014). Camelina produces high seed yields up to over $3000 \mathrm{~kg} \mathrm{ha}^{-1}$ in favorable environments, with growing well on marginal land and the seed yield over other oilseed crops such as flax under drought, cold, saline and low input soil conditions (Zubr, 1997;Petrie et al., 2014). Furthermore, camelina has low requirements, a short life cycle (80-100 days), high

\footnotetext{
*Corresponding author:

Runzhi Li, Institute of Molecular Agriculture and Bioenergy, Shanxi Agricultural University, Taigu 030801, Shanxi, China.

E-mail: rli2001@126.com

Received: 15 October 2016;

Revised: 14 February 2017;

Accepted: 16 February 2017;

Published Online: 26 February 2017
} 
disease-pest resistance and stress tolerance (Zanetti et al., 2013; Zubr, 1997).

Seed oil is predominantly stored in triacylglycerol (TAG), a molecule with three esterified fatty acids in the glycerol backbone. TAG biosynthesis in plants is involved a metabolic pathway consisting of a series of enzymatic reactions through multiple subcellular structures (Wu et al., 2012; Yuan et al., 2015). Briefly, glycerol-3-phosphate (G3P) formed in ER is acylated into lysophosphatidic acid (LPA) by glycerol-3-phosphate acyltransferase (GPAT). LPA is then acylated by lysophosphatidic acid acyltransferase (LPAT) to produce phosphatidic acid (PA). After the two sequential acylations, PA gets dephosphorylated by phosphatidic acid phosphatase (PAP) to generate diacylglycerol (DAG) which is subsequently for final acylation via two different acyltransferases to generate TAGs accumulated in oil droplets in seeds. In Kennedy pathway, diacylglycerol acyltransferases (DGAT) catalizes the final acylation by transfering acyl-CoAs to the $s n-3$ position of DAG to synthesize TAG. However, in acyl-CoA independent pathway, acyl group (the $s n-2$ acyl group) is transferred from phospholipids into DAG by phospholipid: diacylglycerol acyltransferases (PDAT), forming TAG (Ståhl et al., 2004; Dahlqvist et al., 2000, Liu et al., 2012).

There are two distinct groups of DGAT enzymes with no homology to each other, designated DGAT1 and DGAT2. Both them are non-redundantly responsible for the majority of TAG biosynthesis. The contribution of DGAT1, DGAT2 and PDAT for TAG synthesis is varied between species (Li et al., 2013). Although a lot of reports showed that this final acylation catalyzed by each of three classes of enzymes is the rate-limiting step in TAG biosynthesis, little is known about how they are regulated and their functions in other cellular process. TAG synthesis is essential for seed development, and the pathway blockage resulted in disruption in embryonic development (Zhang et al., 2009). Moreover, TAG accumulation in other vegetative tissues was induced by stress factors such as low nitrogen, drought and senescence in Arabidopsis seedlings (Yang et al., 2011; Kaup et al., 2002). However, it remains to be addressed which of the three enzymes above is responsible for such enhance in TAG synthesis under stress conditions.

Here, we use the camelina reference genome released in 2014 (Kagale et al., 2014) to identify members of each family of DGAT1, DGAT2 and PDAT in this genome with $217 \%$ of the expanded lipid gene family compared to Arabidopsis. Quantitative PCR (qPCR) was employed to examine the expression pattern of each member for elucidating roles of the individual member in camelina seed developing and other vegetable tissues, particularly in responses to environmental stresses. Our data shows that DGAT1, DGAT2 and PDAT family members function differently for TAG accumulation and plant responses to cold and salt stresses, providing new knowledge for understanding of regulation mechanism of oil accumulation and the related gene functions in various life activities.

\section{MATERIALS AND METHODS}

\section{Plant material, growth conditions and sampling}

Camelina sativa cultivar "SC-N1" commercially planted for 5 years in Taigu County, Shanxi province, China (E112.32 ${ }^{\circ}, \mathrm{N} 37.26^{\circ}$ ) was selected in this study. The plants were cultivated in the environmental-controled greenhouse at $23{ }^{\circ} \mathrm{C}$ under natural light conditions. The high-pressure sodium lights $\left(250 \mu \mathrm{E} \mathrm{m}^{-2} \mathrm{~s}^{-1}\right)$ were also supplemented. The photoperiod was set as $16 \mathrm{~h}$. Normal managements of watering and fertilization were used for plant cultivation. During period of inflorescence, labeled tags were attached to the emerging flowers. The samples of flowers and seed pods were harvested at 7, 15, 22, 29, and 36 days after flowering (DAF), which were subsequently used for PCR, fresh weight, fatty acid composition, and oil as well as protein accumulation analysis, respectively.

Fully-developed leaves, stems and roots were collected from the 7-cauline leaf plants that were cultivated with half-strength Murashige and Skoog (MS) medium (Phyto Technology Laboratories). For each experiment, tissue samples were harvested from at least 6 camelina plants. All collected samples were immediately frozen in liquid $\mathrm{N}_{2}$ and storaged in $-80{ }^{\circ} \mathrm{C}$ untill total RNA preparison and other experimental measurements.

\section{Stress treatment and sampling}

The same C. sativa cultivar was cultivated at $23^{\circ} \mathrm{C}$ with photoperiod of 16-h light/8-h dark cycle. The 3-week-old soil-grown seedlings were selected for stress experiments. For cold stress treatment, the seedlings were kept at $4^{\circ} \mathrm{C}$ for up to 6 days with a 16-h photoperiod in climatic chamber. Samples were collected at 0, 1, 2, 4, and 6 day under the treatment. For salt stress treatment, the seedlings were irrigated by solutions containing $150 \mathrm{mM} \mathrm{NaCl}$ in climatic chamber. The seedlings were sampled at $0,6,12$, 24 , and $48 \mathrm{~h}$ after the stress. All the samples were frozen immediately in liquid $\mathrm{N}_{2}$, and then used for RNA extraction, oil measurement and other analysis. These experiments were repeated at least six times.

\section{Lipid preparison and fatty acid examination by GC}

Camelina seeds and vegetative tissues were sampled for total lipid extraction using the method described by Xue et al. (2013). In short, 20-30 mg of each sample were taken 
from seeds at different phases of development or vegetative tissues of camelina plants. Under high vacuum, the samples were freeze-dried for $48 \mathrm{~h}$, and then weighed. The water content was calculated by sample weight recorded before freeze-drying minus the weight recorded after freezedrying. The frozen-dried samples were added into glass tube, followed by placing $1 \mathrm{ml} 5 \%(\mathrm{v} / \mathrm{v})$ sulfuric acid in methanol (freshly prepared), $25 \mu 10.2 \%$ butylated hydroxy toluene (BHT)in methanol, $10 \mu \mathrm{g}$ triheptadecanoin (17:0) and $300 \mu \mathrm{l}$ toluene as co-solvent. The mixture (sample + chemical solutions) was vortexed shortly, and then heated at $90-95^{\circ} \mathrm{C}$ for $1.5 \mathrm{~h}$. When the mixture was cooled to room temperature, add $1.5 \mathrm{ml} 0.9 \% \mathrm{NaCl}(\mathrm{w} / \mathrm{v})$ and $1 \mathrm{ml}$ hexane into the mixture for the transmethylation reaction. The homogenate was phase separated by spinning for $5 \mathrm{~min}$ at $2000 \mathrm{rpm}$. The upper organic phase containing fatty acidmethyl esters (FAMEs) was removed to a freshdried tube, while the resulting aqueous phase was extracted with $2 \mathrm{ml}$ hexane, and the phases were separated again by centrifugation. The organic phases were combined, followed by during with a flow of $\mathrm{N}_{2}$ gas. The almost dried extracts were then dissolved in $1 \mathrm{ml}$ hexane and $20 \mu \mathrm{l}$ of the sample was transferred to vials used specially for gas chromatography (GC) analysis.

The FAMEs of sample lipids were separated by GC analyzer (Aglient7890B) using a $0.25 \mathrm{~mm}$ i.d. $\times 0.33 \mu \mathrm{m} \times$ $10 \mathrm{~m}$ HP-88 column and quantified using a flame-ionization detector. The GC experiments were performed using three biological replicate samples. The fatty acid compositions were identified by comparison of their retention times with those of the known standards. The oil content in sample was quantified by comparing the concentration of the fatty acids to the peak areas of the added internal standard (the concentration is known). All data were analyzed statistically. The $t$-test was used to determine the significance of difference between pair-wise means.

RNA preparison, cDNA synthesis and qPCR examination Total RNA derived from each sample was isolated with plant RNeasy mini kit (Sigma-Aldrich) according to the manufacturer's instructions. RNA samples extracted were further treated by DNaseI (Promega) to remove contaminating DNA. NanoDrop 2000 spectrophotometer (Thermo Scientific) was employed to measure RNA concentrations $(\mathrm{ng} / \mu \mathrm{l})$ and purity ratios $(260 / 280 \mathrm{~nm}$ and $260 / 230 \mathrm{~nm})$.

Five $\mu \mathrm{g}$ of total RNA prepared from each sample was taken as the template for the first strand cDNA synthesis using a First-Strand cDNA Synthesis Kit (Fermentas). The manufacturer's procedure was employed for real-time PCR experiments with a cycling program of $42{ }^{\circ} \mathrm{C}$ for $30 \mathrm{~min}$ in each cycle. The cDNA level in each sample was quantified, and then used as the templates by diluting to a final concentration of $100 \mathrm{ng} / \mu \mathrm{l}$ for qRT-PCR analysis.

All qRT-PCR were carried out in an iCycleriQ detection system (Bio-Rad) with. SYBR Green I Master Mix kit (Applied Biosystems). The reaction mixture without added reverse transcriptase was used as the PCR controls to ensure RNA samples were free of DNA contamination. PCR reaction for each sample was performed in triplicate for three biological replicates. The $25-\mu l$ volumes of reaction system included $1 \mu l$ of each forward and reverse primer $(500 \mathrm{nM}), 12.5 \mu \mathrm{l}$ of SYBR green master mix, $5 \mu \mathrm{l}$ of a 1:10 (v/v) dilution of cDNA, and $5.5 \mu \mathrm{l}$ of HPLC molecular biology grade water. PCR reactions were proceed in MicroAmp 96-well plates (Applied Biosystems) covered with optical adhesive covers (Applied Biosystems). PCR program was set as the followings: initial activation at 95 ${ }^{\circ} \mathrm{C}$ for $10 \mathrm{~min}$, followed by a total of $30-40$ cycles with each containing a two temperature thermal cycle (denaturation at $95^{\circ} \mathrm{C}$ for $15 \mathrm{~s}$, and annealing extension at $60^{\circ} \mathrm{C}$ for $1 \mathrm{~min}$ ), and a final extension at $72^{\circ} \mathrm{C}$ for $5 \mathrm{~min}$.

The $2^{-\Delta \Delta C t}$ calculation method (Schmittgen et al., 2008) was conducted to quantify PCR products. The $\beta$-actin gene of camelina was used as internal control to normalize the relative amount of the target $\mathrm{mRNAs}$ tested. The error bars represent the standard errors (SE) for the fold changes of relative expression of the target genes. The gene expression amount was calculated from three independent biological replicates and triplicate PCR reactions for each sample. The primers of the interested genes used for qRT-PCR analysis are listed in Table 1.

\section{RESULTS}

Identification of DGAT1, DGAT2 and PDAT family members in camelina genome

Arabidopsis AtDGAT1 (AT2G19450), AtDGAT2 (AT3G51520) and AtPDAT (AT5G13640) protein were selected as query sequences to perform BLASTP search against the annotated genome of Camelina sativa (https:/ / www.ncbi.nlm.nih.gov/genome/?term=camelina+sativa), respectively, leading to the identification of nine predicted proteins highly identical $(>90 \%)$ to the corresponding Arabidopsis homologs (Table 2). Three members were identified for each family of DGAT1, DGAT2 and PDAT in camelina genome, respectively, sharing very high amino acid sequence similarity $(>95 \%)$ among three members in a family.

Camelina CsDGAT1 family contains three members namely as CsDGAT1-A (XP_010415368), CsDGAT1-B (XP_010489480), and CsDGAT1-C (XP_010468809), 
Table 1: Primer sequences used for PCR analysis on the target genes in $C$. sativa

\begin{tabular}{lll}
\hline Gene & Primer sequences & Reverse primer (5'-3') \\
\cline { 2 - 3 } & Forward primer (5'-3') & ATGGCTCTGTTTGAAGATTGC \\
\hline CsDGAT1-A & CACCCTCTGATGATGTTGG & GCATACGTAGTCTCGGCGTT \\
CsDGAT1-C & CGAGATCGGATTCCAACGGA & TTTCGTTATCCCCGGCCAAA \\
CsDGAT2-A & TTTTGGATCTGGAGGCGGC & CCAAAGACATAGGCACGA \\
CsDGAT2-B & CGCCATTCACTTCAACAT & TCGGGATGAAGATAAACAGA \\
CsDGAT2-C & AAGCGGACAAACCAAAGA & AAAGACATAGGCACGATT \\
CsPDAT1 & CGCCATTCACTTCAACAT & CACGACCACTTCCCTCCT \\
CsPDAT2 & GTCTAATCTGGGTTCTTCC & AGCTCAAGCCCACCAGTAAC \\
CsPDAT3 & AGAAATCGAACGGAGGAGGC & CGGGTAAAGGACCCGTGATT \\
ß-actin & TCTGAAGAGGTGGTGAACGC & AGGGTTCTCTCTTCCACATGCCA \\
\hline
\end{tabular}

Table 2: Identification of DGAT1, DGAT2 and PDAT family members in camelina genome

\begin{tabular}{|c|c|c|c|c|c|c|c|}
\hline Gene & Gene ID & Chrom-osome & Location (NC_) & $\begin{array}{c}\text { mRNA accession } \\
\text { (XM_) }\end{array}$ & mRNA size (bp) & $\begin{array}{c}\text { Protein } \\
\text { accession }\left(X_{2}\right)\end{array}$ & $\begin{array}{l}\text { Protein } \\
\text { size (aa) }\end{array}$ \\
\hline CsDGAT1-A & 104701387 & 1 & 025685.1 & 010417066.1 & 1885 & 010415368.1 & 520 \\
\hline CsDGAT1-B & 104767141 & 19 & 025703.1 & 010491178.1 & 2069 & 010489480.1 & 520 \\
\hline CsDGAT1-C & 104748933 & 15 & 025699.1 & 010470507.1 & 2030 & 010468809.1 & 474 \\
\hline CsDGAT2-A & 104711698 & 9 & 025693.1 & 010428422.1 & 1299 & 010426724.1 & 315 \\
\hline CsDGAT2-B & 104791388 & 6 & 025690.1 & 010517268.1 & 1376 & 010515570.1 & 315 \\
\hline CsDGAT2-C & 104780989 & 4 & 025688.1 & 010505546.1 & 1344 & 010503848.1 & 315 \\
\hline CsPDAT1 & 104735371 & 13 & 025697.1 & 010455150.1 & 2461 & 010453452.1 & 671 \\
\hline CsPDAT2 & 104705618 & 8 & 025692.1 & 010421655.1 & 2456 & 010419957.1 & 597 \\
\hline CsPDAT3 & 104769582 & 20 & 025704.1 & 010493830.1 & 2496 & 010492131.1 & 671 \\
\hline
\end{tabular}

with protein size consisting of 520,510, and 474 amino acids, respectively. For camelina CsDGAT2 family, three members were designated CsDGAT2-A (XP_010426724), CsDGAT2-B (XP_010515570), and CsDGAT2-C (XP_010503848), with the same protein size (315 amino acids). Three members of CsPDAT family were termed CsPDAT1 (XP_010453452), CsPDAT2 (XP_010419957), and CsPDAT3 (XP_010492131), containing 671, 597, and 671 amino acids in length, respectively.

Phylogenetic analysis was conducted for these camelina proteins examined here plus a number of DGAT1, DGAT2 and PDAT proteins from other plant species (Fig. 1). All 24 target protein sequences were clearly separated into DGAT1, DGAT2 and PDAT families with extremely strong bootstrap support. The three members of each camelina family tested here were clustered closely within their family groups, further confirming that these camelina proteins obtained are identical proteins of DGAT1, DGAT2 and PDAT families.

\section{Fattyacid composition and oil accumulation in camelina seed development and other tissues}

The seed development of Brassicaceae plants generally can be divided into the initial "growth phase", the second "accumulation phase", and the final "desiccation phase". "Growth phase" begins at the end of fecundation to young embryonic formation, with rapid cell division but little deposition of storage material. In "accumulation phase", embryo continues growing, and storage oil and protein are synthesized rapidly. The "desiccation phase" is characterized by dehydration and little synthetic activity. Here, for better examining dynamical changes of oil and fatty acid accumulation, camelina seed development is divided into five stages (Fig. 2) based on size and color of seed and silique, seed fresh weight, and days after flowering (DAF).

FAME (fatty-acid methyl esters) analysis was employed on camelina seed sampled at different development stages of plants grown in the greenhouse. Our results showed that oil accumulation was to be a continuous increase pattern like "S" curve throughout seed development (Fig. 3). Notably, the rate of oil accumulation was varied at different development stages. The lower rate $3 \%$ increase per day) was observed in initial growth phase (between Stage 1 and 2), followed by higher rate $(1.9 \%$ increase per day) in accumulation phase (from Stage 2 to Stage 4$)$, and then little lower rate ( $\sim 0.5 \%$ increase per day) in final desiccation phases (from Stage 4 to maturation) and total oil peak of $34.9 \%$ (w/dry weight) at Stage 5. Accompanying this dynamic change of oil accumulation, protein deposit was also to enhance progressively during seed development, with a rapid elevation $(0.8 \%$ increase per day) between Stage 1 to Stage 3, and then a slow increase $(0.4 \%$ increase per day) from Stage 3 through 


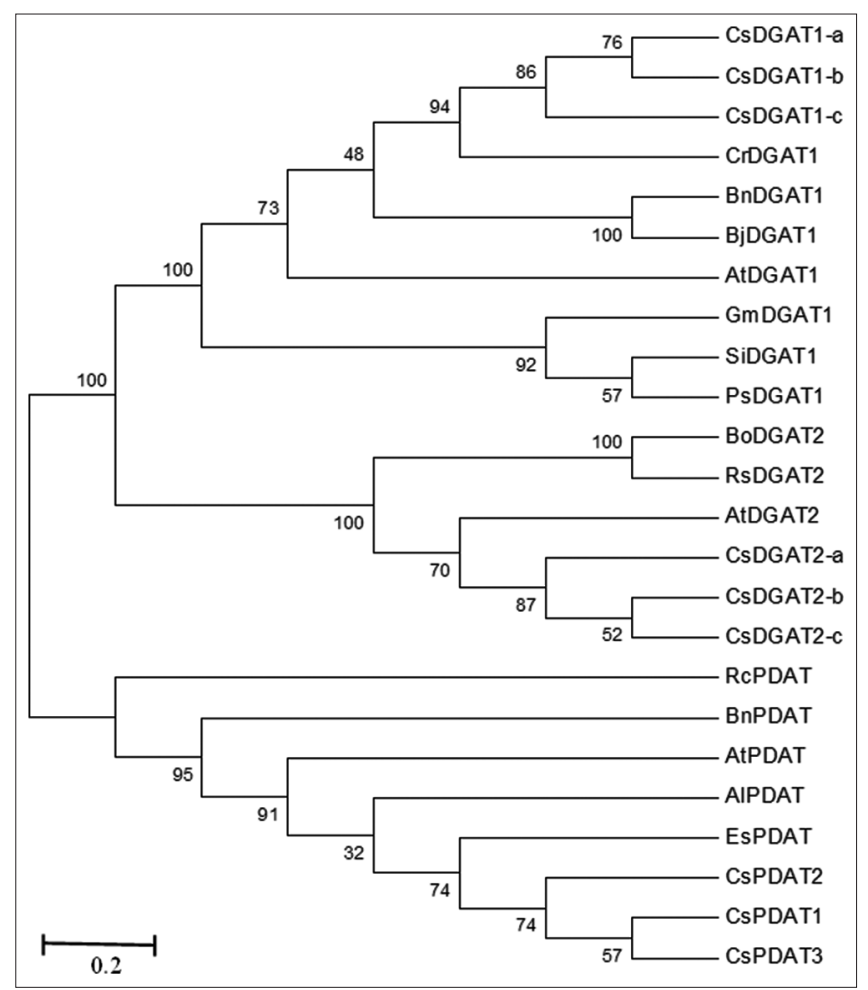

Fig 1. The phylogenetic analysis based on protein sequences of DGAT1, DGAT2 and PDAT from $C$. sativa and other plant species. The analysis was performed using the MEGA 6.0 program with neighbor joining method and with 1000 replicates. Numbers are bootstrap values. The 0.2 scale represents $20 \%$ change, calculated as estimated numbers of replacements. The protein sequences used here were AIPDAT (XP_002871577) from Arabidopsis lyrata; AtDGAT1 (AT2G19450), AtDGAT2 (AT3G51520) and AtPDAT (AT5G13640) form Arabidopsis thaliana; BjDGAT1 (AAY40784) from Brassica juncea; BoDGAT2 (XP_013602347) from Brassica oleracea; BnDGAT1 (CDY69607) and BnPDAT (CDX85636) from Brassica napus; CrDGAT1(XP_006299296) from Capsella rubella; EsPDAT (XP_006399859) from Eutrema salsugineum; GmDGAT1 (NP_001242457) from Glycine max, PsDGAT1 (AIX97817) from Prunus sibirica; RcPDAT: (NP_001310694) from Ricinus communis; RsDGAT2 (XP_018470674) from Raphanus sativus; SiDGAT1 (NP_001291334) from Sesamum indicum. GenBank accession numbers are provided in parentheses. Accession numbers for all members of camelina CsDGAT1, CsDGAT2 and CsPDAT were listed in Table 2.

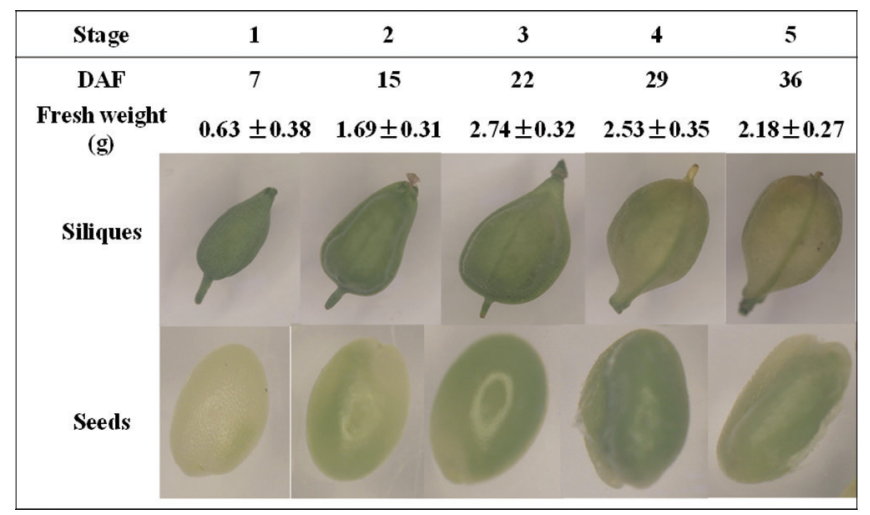

Fig 2. Five development stages of $C$. sativa seeds and siliques. The development is divided into five stages based on size and color of seed and silique, seed fresh weight, and days after flowering (DAF), fresh weight represents the mean \pm SE level of five independent replicates. maturation at which total protein content was up to $22.8 \%$ (w/dry weight).

Fatty acid profiles also exhibited the developmental stage-specific changes in the fatty-acid concentration (Fig. 4). Eight main fatty acids were detected in camelina seed, including palmitic acid (16:0), stearic acid (18:0), oleic acid (18:1), linoleic acid (18:2), linolenic acid (18:3), arachidic acid (20:0), eicosenoic acid (20:1), and erucic acid (22:1). The profiles of these fatty acids were almost contrary between the early stages (up to $15 \mathrm{DAF}$ ) of seed development and the mature seeds. Early stages showed high levels of palmitic $(21.9 \%$ at $7 \mathrm{DAF})$, stearic $(20.4 \%$ at $7 \mathrm{DAF})$, oleic $(22.1 \%$ at $15 \mathrm{DAF})$, and linoleic acid $(34.3 \%$ at $15 \mathrm{DAF})$, whereas mature seeds contain high levels of $\alpha$-linolenic and eicosenoic acid (41.3\% and 12.2 $\%$ ). As seed development, contents of polyunsaturated linolenic, eicosenoic (start at $22 \mathrm{DAF}$ ), and erucic (start at $22 \mathrm{DAF}$ ) acids increased largely, and peaked at mature stage. Accordingly, accumulations of three saturated palmitic, stearic, and arachidic (start at $15 \mathrm{DAF}$ ) acids decreased progressively, reaching their lowest levels in mature seeds. Moreover, a significant reduction was observed for monounsaturated oleic acid (start at $15 \mathrm{DAF}$ ) and polyunsaturated linoleic acid (start at $22 \mathrm{DAF}$ ) throughout seed development.

Analysis of oil content and fatty acid profiles were also conducted for camelina vegetable tissues including root, stem and leaf of 7-cauline leaf plants. As shown in Fig. 5, compared to seed, total oil content was very lower in vegetable tissues, with $5.1 \%$ in leave, $3.8 \%$ in root and $4.5 \%$ in stem. All eight main fatty acids in seed were detected in camelina plant vegetable tissues, with varied contents in the tissues tested (Fig. 6). The most abundance of fatty acid is linolenic acid with $29.7 \%$ in roots, $37.3 \%$ in stems and $42.4 \%$ in leaves, respectively, followed by linoleic acid accounted for $19.7 \%$ in root, $18.3 \%$ in stem and $10.6 \%$ in leaves. Saturated palmitic acid was the third high fatty acid in vegetable tissues with $16.4 \%$ in roots, $19.2 \%$ in stems and $17.7 \%$ in leaves. Additionally, high level of $16: 3$ was detected in leaves $(13.5 \%)$, photosynthesis organs while only trace level $(<1 \%)$ of 16:3 in other non-photosynthesis organs such as roots and stems. Compared with seeds, vegetable tissues contained lower levels $(<5 \%)$ of very long chain fatty acids (VLCFAs) such as arachidic (20:0), eicosadienoic (20:1), and erucic (22:1) acids.

\section{Expression patterns (dynamics) of members of CsDGAT1, CsDGAT2 and CsPDAT in camelina seed development and other tissues}

Quantitative RT-PCR (qPCR) was employed to examine the steady-state mRNA levels of nine target genes encoding the above members of CsDGAT1, CsDGAT2 and Emir. J. Food Agric • Vol $29 \bullet$ Issue 4 • 2017 


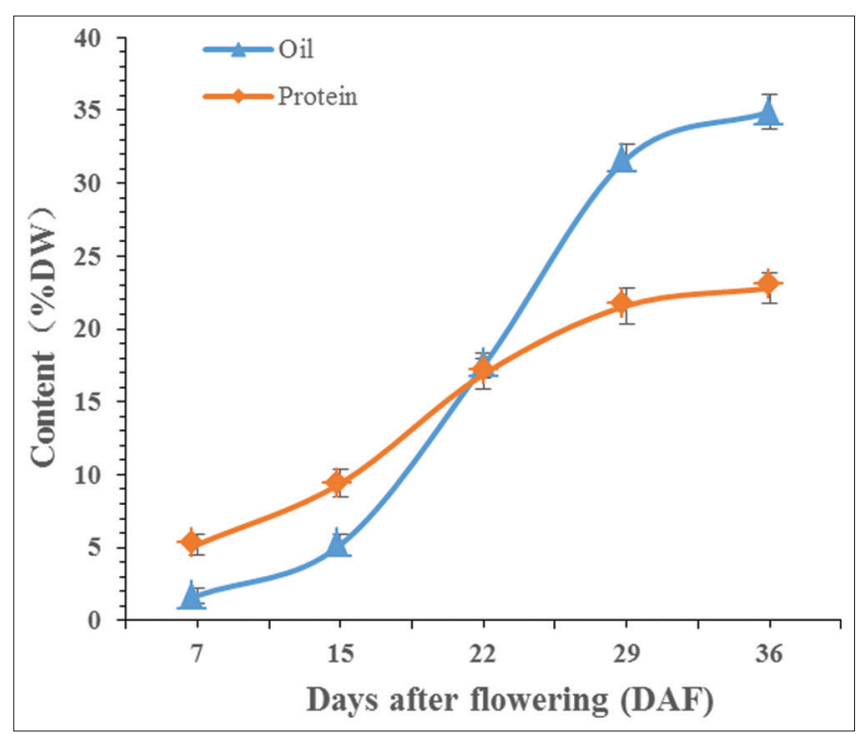

Fig 3. Oil and protein accumulations in $C$. sativa seeds at five different development stages. Data represents the average of five independent replicates, and the error bars represents SE.

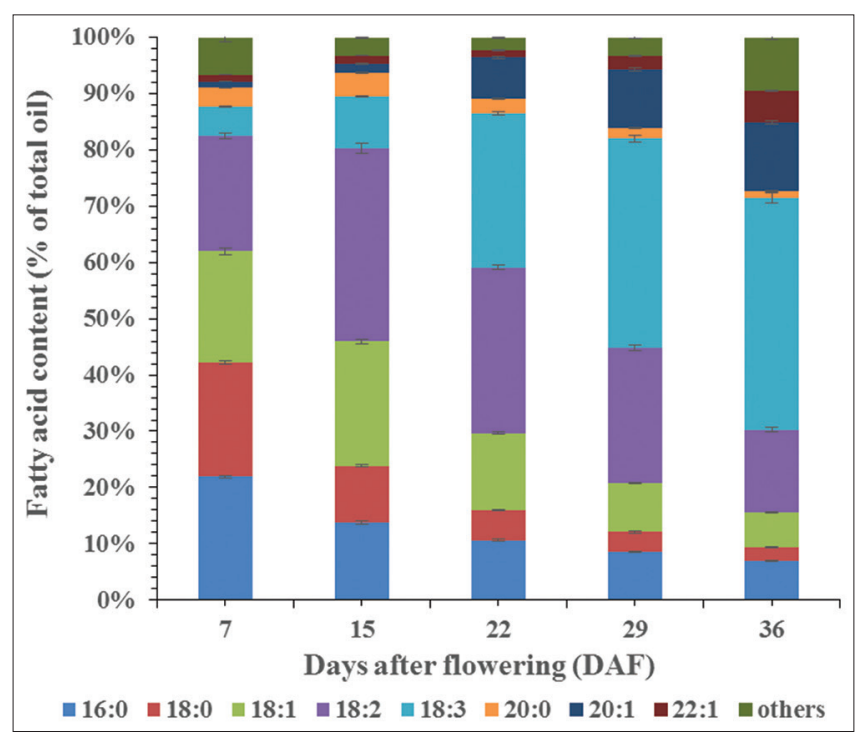

Fig 4. Changes of fatty acid composition during camelina seeds development. (16:0): palmitic acid, (18:0): stearic acid, (18:1): oleic acid, (18:2): linoleic acid, (18:3): linolenic acid, (20:0): arachidic acid, (20:1): eicosenoic acid, (22:1): erucic acid. Fatty acid composition in developing seeds were tested as described in materials and methods. Data represents the average of five independent replicates, and the error bars represents SE.

CsPDAT families, respectively, in various camelina tissues including root, stem, leaf, flower and seed at different development stages $(7,15,22,29$, and $36 \mathrm{DAF})$. Different expression patterns were detected for those genes in different tissues tested. For developing camelina seeds, mRNAs of CsDGAT1s (mainly CsDGAT1-A) expressed at large abundance (Fig. 7a), followed by CsDGAT2s (mainly CsDGAT2-C) (Fig. 7b), with CsDGAT1-A transcripts higher than $C s D G A T 2-C$ by two times. On

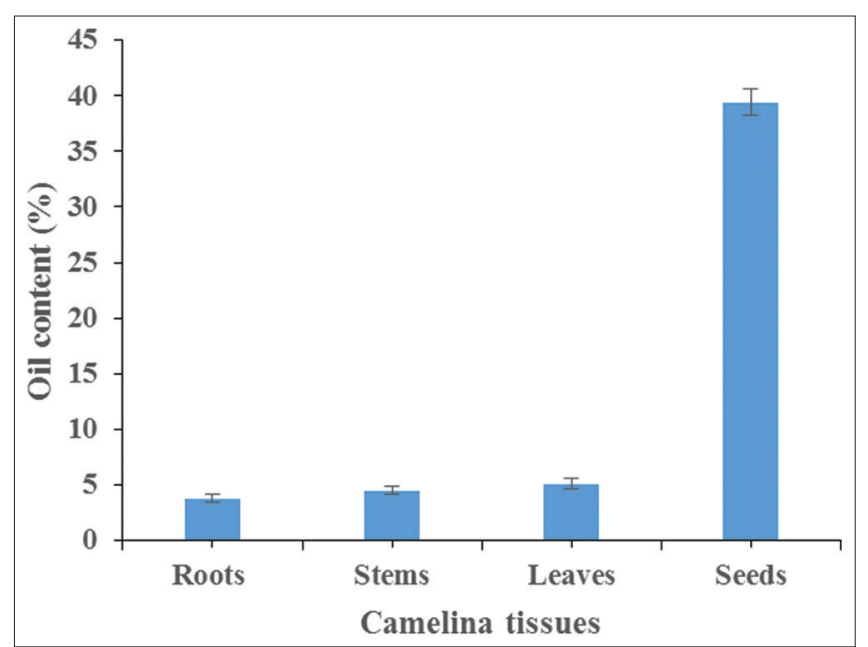

Fig 5. Oil contents in different tissues of $C$. sativa. Oil contents were measured as described in materials and methods. Data represents the average of five independent replicates, and the error bars represents SE.

other hand, only a trace level of CsPDAT mRNAs was detected in seed (Fig. 7c). Particularly, the tremendous increasing of CsDGAT1-A and CsDGAT2-C expression appeared in seeds between 15 and $22 \mathrm{DAF}$, the period for vast enhancement of oil accumulation, suggesting that CsDGAT1-A and CsDGAT2-C contribute oil accumulation in camelina seeds with CsDGAT1-A much important.

CsPDAT3 expression was extremely high in leaves, and moderate amount in flowers (Fig. 7f), followed by medium level of CsDGAT1-C transcripts in both tissues (Fig. 7d), indicating that CsPDAT3 and CsDGAT1-C function importantly for oil accumulation and possible other lipidrelated processes taking place in those tissues. For camelina roots and stems, CsDGAT2-A expressed at a considerable level (Fig. 7e) while very low transcripts of the rest eight member genes detected, demonstrating that CsDGAT2-A is active for oil biosynthesis in toot and stem tissues.

Differential expressions of CsDGAT1, CsDGAT2 and CsPDAT members induced by cold and salt stresses To determine whether members of CsDGAT1, CsDGAT2 and CsPDAT involve in camelina response to abiotic stresses, 3-week-old seedlings grown under cold or salt stress were harvested, respectively. The seedling samples were separated into two batches: one for total lipid extraction and TAG measurement, the other for total RNA extraction and qRT-PCR assay.

The amounts of TAGs in seedlings were gradually increased during cold stress with dramatic enhancement appearing from $4 \mathrm{~d}$ to $6 \mathrm{~d}$ under the treat. The peak level of TAGs in the cold-stressed samples was more than two times higher than that in the control (Fig. 8). Similarly, TAG 


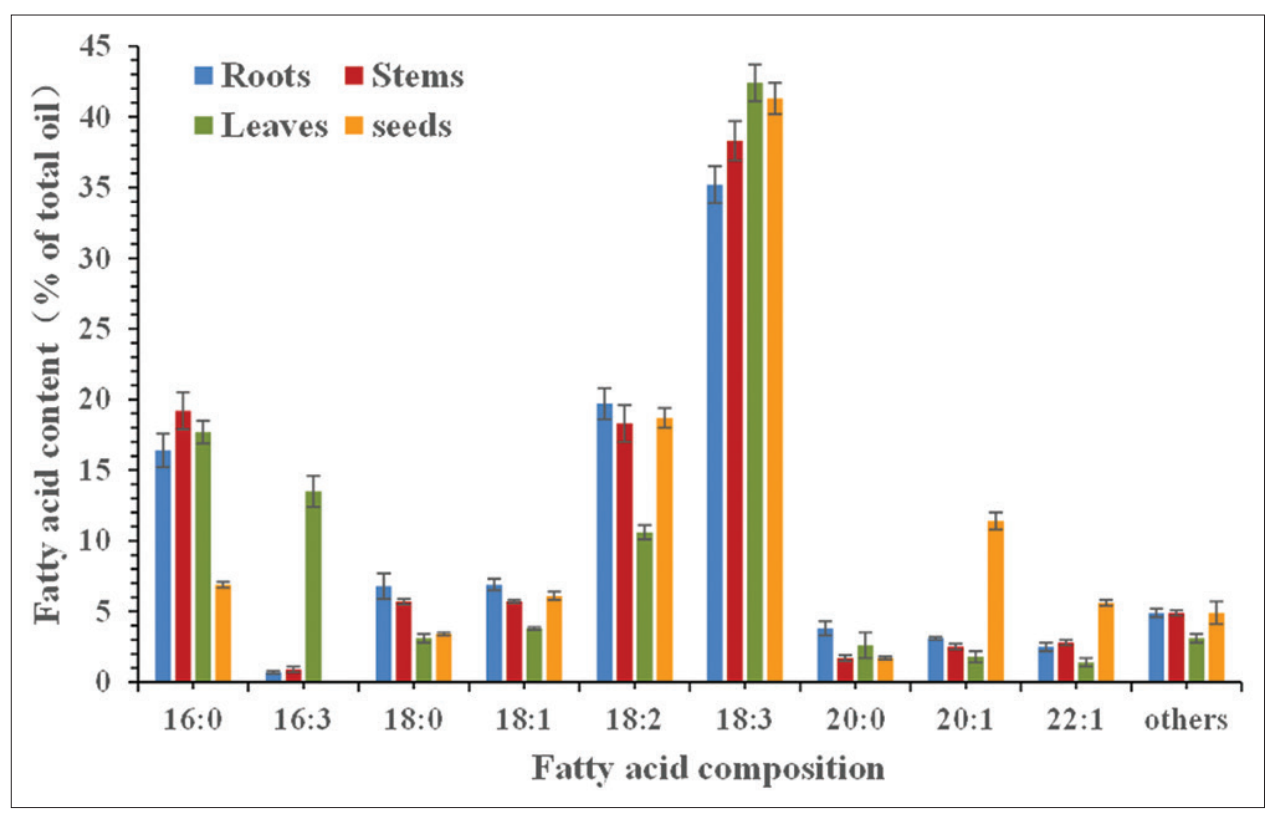

Fig 6. Fatty acid profiles in different tissues of C. sativa. (16:0): palmitic acid, (18:0): stearic acid, (18:1): oleic acid, (18:2): linoleic acid, (18:3): linolenic acid, (20:0): arachidic acid, (20:1): eicosenoic acid, (22:1): erucic acid. Fatty acid composition in developing seeds were tested as described in materials and methods. Data represents the average of five independent replicates, and the error bars represents SE.

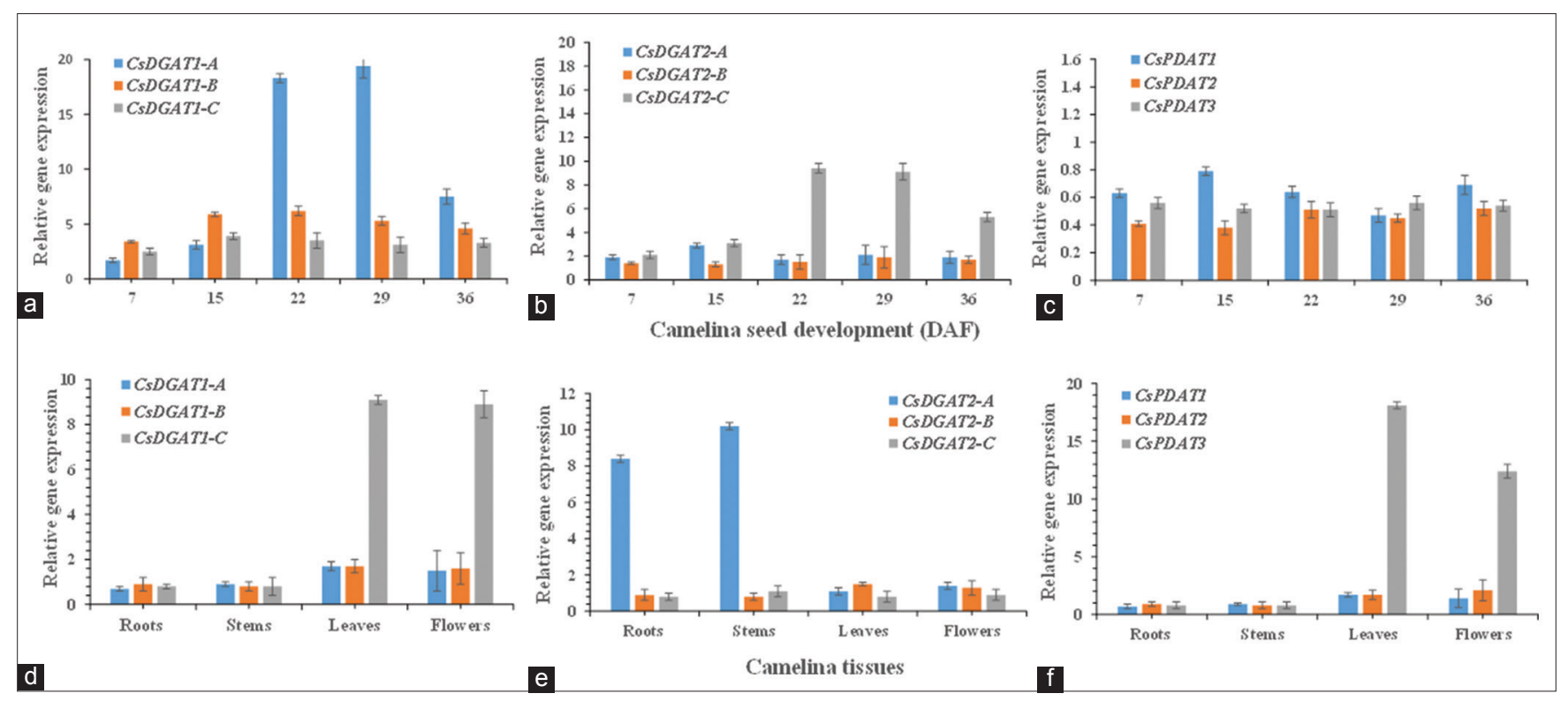

Fig 7. Expression profiling of CsDGAT1, CsDGAT2 and CsPDAT in different tissues of $C$. sativa (a) CsDGATs, (b) CsDGAT2s and (c) CsPDATs in seed development; (d) CsDGATs, (e) CsDGAT2s and (f) CsPDATs in root, stem, leaf and flower tissues. Expressions were examined by quantitative real-time PCR in different camelina tissues. Gene expression levels were normalized with respect to the internal control $\beta$-actin gene. Data bars represent the mean \pm SE level of relative transcript abundance of six replicates.

accumulation increase progressively up to maximum level (1.9 folds higher than the control) at $24 \mathrm{~h}$ in the presence of salt treat, with rapid increase occurring from $12 \mathrm{~h}$ to $24 \mathrm{~h}$ under the stress (Fig. 8).

Differential expressions of CsDGAT1s, CsDGAT2s and CsPDATs were observed in camelina seedlings induced by stresses. Cold stress greatly upregulated the expression of CsDGAT2-B by 11.6 folds compared to the control, with rapid increase present between $2 \mathrm{~d}$ and $4 \mathrm{~d}$ after the treat (Fig. 9b). Another cold-upregulated gene is $C s P D A T /$ whose peak level (in $6 \mathrm{~d}$ ) lower than that of CsDGAT2-B (in $6 \mathrm{~d}$ ) by almost 2 folds (Fig. 9c). Other members of these gene families tested showed no obvious changes during cold stress in comparison to the control (Fig. 9a-c).

For salt stress, transcript levels of CsDGAT1-B and CsPDAT2 were massively upregulated up to their peaks 
at $12 \mathrm{~h}$ and $24 \mathrm{~h}$, respectively, with 15 folds and 8.5 folds higher than the control, respectively (Fig. 9d, f). However, CsPDAT3 and CsDGAT1-C, CsDGAT2-A mRNAs were declined during the stress (Fig. 9d, e, f). Other gene members tested was not changed significantly.

Overall, dynamic analysis above evidence that $C s D G A T 2-B$ and CsPDAT1 expressions were coincides with the increase of oil accumulation under cold stress, whereas CsDGAT1-B and CsPDAT2 expressions were closely associated with the elevation of oil amount induced by salt stress.

\section{DISCUSSION}

Plant seed oils are very important source for human fatty acid nutrition, also serving as the excellent hydrocarbon chains for industrial products. With oil enriched in $\omega-3$ fatty acids and several excellent agronomic performances, camelina became a very important oilseed for sustainable production of high-valued oils with wide applications (Kagale et al., 2014; Yuan et al., 2015; Haslam et al., 2016). Like other oil crops, camelina seeds produce high level of oils with TAG as the predominantly stored form used as the major carbon and energy reserve during plant life cycle. It is much needed to explore the regulatory mechanism of TAG biosynthesis in seeds and other tissues, particularly under abiotic stresses, providing valued knowledge for understanding lipid metabolism and higher tolerance to stresses, and benefiting camelina breeding of new varieties with high yield, healthy quality and wide adaptation. Here, we take DGAT1, DGAT2 and PDAT, three key enzymes active at the final acylation in TAG biosynthesis as the targets for this investigation.

Our data showed all three gene families present in camelina genome. Moreover, each family has three members sharing
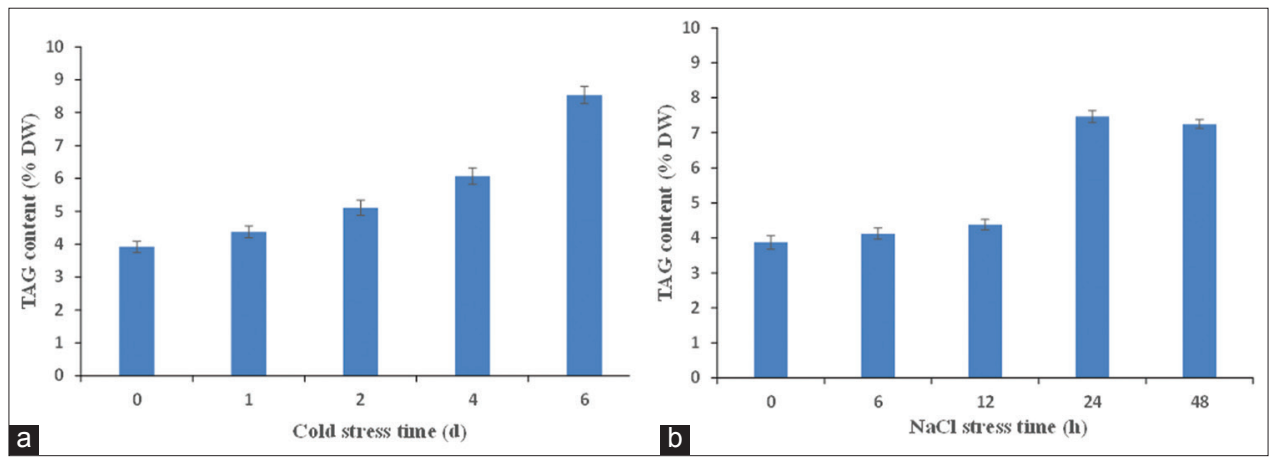

Fig 8. TAG accumulation in camelina seedlings induced by cold and salt stresses. (a) Samples were collected at $0,1,2,4$, and 6 day under the cold stress treatment. (b) Samples were collected at 0, 6, 12, 24, and $48 \mathrm{~h}$ after the salt stress treatment. Data represents the average of five independent replicates, and the error bars represents SE.

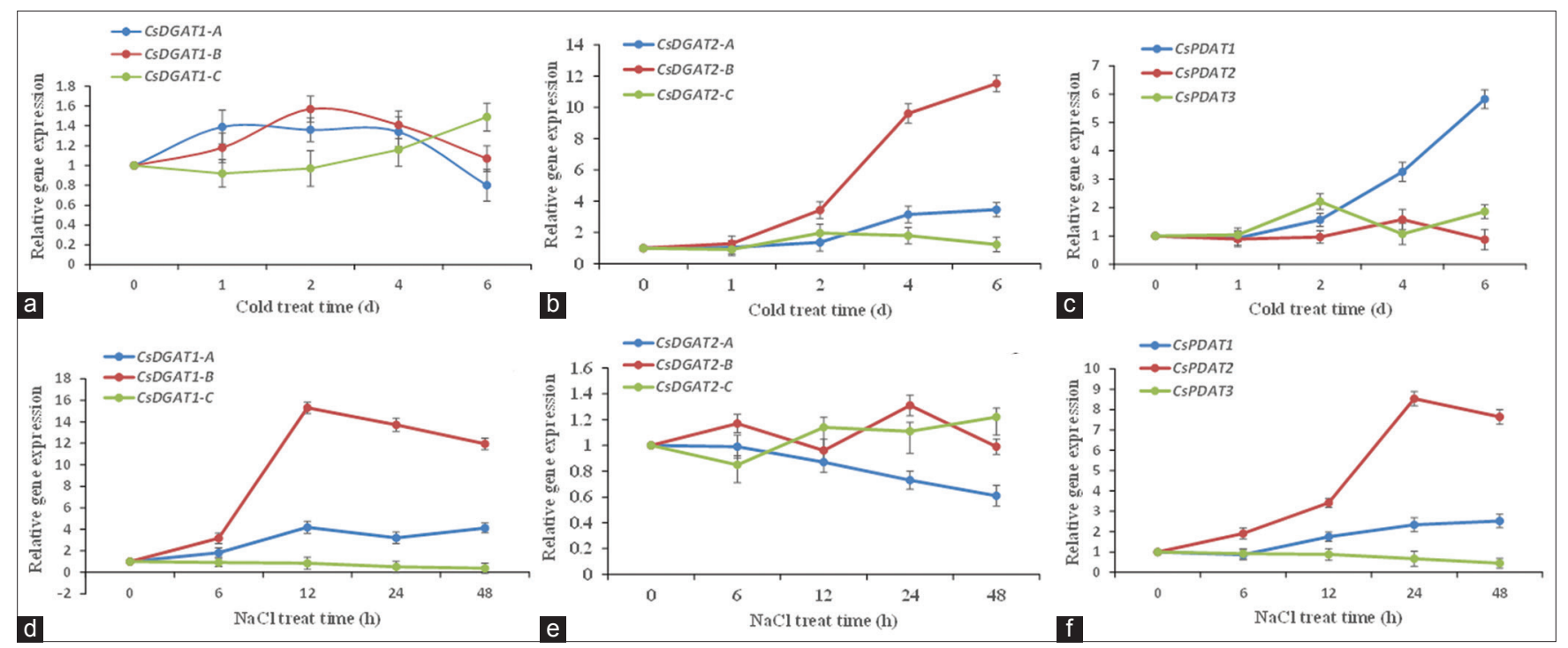

Fig 9. Differential expressions of CsDGAT1s, CsDGAT2s and CsPDATs induced by cold (a, b, c) and salt (d, e, f) stresses in C. sativa. Expressions profiles were examined by qRT-PCR in camelina seedling under stresses. The quantification of the genes is measured relative to the expression of the indigenous housekeeping gene $\beta$-actin. The control sample expression was used as the calibrator (set as 1 ) for the remaining samples. Error bars represent the standard error \pm SE of six biological replicates. 
a very high identity to each other (Table 2, Fig. 1), unlike in model plant Arabidopsis and some diploids where both DGAT1 and DGAT2 contain a single member. Based on their homology with the corresponding Arabidopsis ortholog, the members identified for the three gene families in camelina genome were designed CsDGAT1-A (XP_010489480), CsDGAT1-B (XP_010489480), CsDGAT1-C (XP_010468809), CsDGAT2-A (XP_010426724), CsDGAT2-B (XP_010515570), CsDGAT2-C (XP_010503848), CsPDAT1(XP_010453452), CsPDAT2 (XP_010419957), and CsPDAT3 (XP_010492131), respectively. This feature of three members is consistency with the findings that camelina genome is highly undifferentiated polyploidy where each gene has more than one copy at least (Kagale et al., 2014). Multiple members for DGAT1, DGAT2 and PDAT were also reported in other oil crops although detail function for each member remains to address. For example, soybean contains 3, 5, and 6 members for DGAT1, DGAT2, and PDAT, respectively (Li et al., 2013).

To investigate the function of each member identified here, we examined the expression patterns of these members in camelina seed development and other tissues including flower, root, stem and leaf. Various expression patterns were detected for these gene members in different tissues (Fig. 7). During seed development (Fig. 7a-c), expressions of CsDGAT1-A and CsDGAT2-C increased greatly, with CsDGAT1- $A$ as bell-shaped and CsDGAT2-C as flat-rise models (Fig. $7 \mathrm{a}$ and b). However, other members displayed a low background expression in seed tissues at one or two stages. Notably, the period of rapid increase expression of the two members is positively associated with the vast enhancement of oil accumulation in developing seeds, indicating both CsDGAT1-A and CsDGAT2-C are major players in TAG assembly and accumulation in camelina seed with CsDGAT1-A much important. This supports previous reports that DGAT1 and/or DGAT2 play critical roles in TAG synthesis in plant seeds, but the contribution varied among species (Jako et al., 2001; Li et al, 2010; Li et al., 2013). Our data further showed which individual member from the three families works predominantly for oil accumulation in seeds. This is of important question need to address, particularly for polyploidy plants like camelina with multiple members in each family of DGAT1, DGAT2 and PDAT.

Similarly, the significant expression was observed for both CsPDAT3 and DGAT1-C mRNAs in flower tissues and growing leaves (Fig. $7 \mathrm{~d}$-f) with $C s P D A T 3$ much higher, showing their involvement in TAG biosynthesis or other lipid-related metabolism occurring in these non-seed organs. The present data is in agreement with the previous reports that DGAT1 and PDAT showed overlapping functions for TAG biosynthesis in Arabidopsis, and they are critical for seed and normal pollen development (Zhang et al., 2009). Furthermore, such expression pattern of CsPDAT3 in camelina leaf and flower was detected for PDAT in Arabidopsis (Stahl et al., 2004) and castor bean (Ricinuscommunis) (Kim et al., 2011), supporting that PDAT functions specially for maintaining membrane lipids in both plant seeds and vegetative organs (Kim et al., 2011). In addition, $C s D G A T 2-A$ was found to express mainly in camelina stem and root, whereas mRNAs of the other members tested just accumulated very low abundance. Collectively, our data displayed that different members of DGAT1, DGAT2 and PDAT families have their own main expression tissues, and possibly function differently.

As discussed above, TAG is typically formed and stored in seed, and also biosynthesized in other vegetative tissues. Interestingly, a number of reports showed that stress could induce TAG accumulation in vegetative tissues of model plant Arabidopsis (Lu et al., 2003; Yang et al., 2011; Petrie et al., 2014). However, it remains to address whether stressinduced TAG accumulation results from newly biosynthesis of TAG or incomplete degradation of the storage oil. If the increased accumulation of TAG is come from the newly TAG biosynthesis, what gene or enzymes are responsible for this TAG biosynthesis under stress condition?

In this study, we found that both cold and salt stresses greatly induced TAG accumulation in camelina seedlings by two folds at least compared to the control (Fig. 8), which coincides with the expression profiles of the gene associated with TAG biosynthesis investigated here. We observed that both CsDGAT2-B and CsPDAT1 expressions were upregulated by 11 and 6 folds (Fig. 9a-c), respectively, demonstrating both members actively function in new TAG biosynthesis under the stress. However, salt stress induced CsDGAT1-B and CsPDAT2 mRNAs upregulated by 15 and 8 folds compared to the control, respectively (Fig. 9d-f), suggesting that CsDGAT1-B and CsPDAT2 mainly work for the new accumulation in camelina seedlings under the stress.

The known reports showed that only AtDGAT1 was found to increase expression, and subsequently catalyzed TAG formation under several stresses in Arabidopsis seedlings (Kong et al., 2013; Yang et al., 2011; Lu et al., 2003). Our findings uncovered that CsDGAT1-B, CsDGAT2-B, CsPDAT1 and CsPDAT2 all participated in the new TAG biosynthesis under stress, but different members were actived for different stress responses in camelina seedlings. These findings demonstrate that newly biosynthesis is the primary source of TAG enhancement induced by stress. Such increase of TAG levels subsequently benefits plant to adapt diverse conditions. 


\section{CONCLUSIONS}

In conclusion, the current study uncover that three members are present in camelina genome respectively for CsDGAT1, CsDGAT2 and CsPDAT protein families, which catalyze the final acylation step in TAG biosynthesis. The expressions of those members display different spatio-temporal patterns. CsDGAT1-A and CsDGAT2-C transcripts enormously accumulate in seed development while $C s P D A T 3$ and $C s D G A T 1-C$ predominantly express in leaf and flower tissues, indicating that different members work at different tissues. Moreover, these members are differentially regulated by abiotic stresses. CsDGAT2-B and $C s P D A T 1$ are upregulated largely in camelina seedlings by cold stress, whereas CsDGAT1-B and CsPDAT2 are upregulated greatly by salt stress, providing the first evidence showing that plant seedlings enhance TAG accumulation to promote adaptation to unfavorable conditions by activation of different members of CsDGAT1, CsDGAT2 and CsPDAT under different stresses. Since camelina oils are desirable resources for human heathy food and bioenergy, the present data would be useful to develop camelina plants with higher biomass yield and increased tolerance against diverse environmental stresses.

\section{ACKNOWLEDGEMENTS}

This work was financially supported by grants from the National Natural Science Foundation (Grant Nos. 30971806; 31201266; 31401430), National “948” Program (Grant No. 2014-Z39), Coal-based Key Sci-Tech Project of Shanxi Province (Grant No. FT-2014-01), University Sci-Tech Innovation Project of Shanxi Province (Grant No. 2016171), and Basic Research for Application Project of Shanxi Province (Grant No. 201601D202060).

\section{Author's contributions}

R. L.: conceived the study and reviewed manuscript. L.Y.: performed the experiments and date analysis. K.Z.: carried out material preparation. Y.S. and C.J.: helped with date analysis. X.M. and J.X.: helped in lipid analysis and manuscript editing. All authors have been involved in drafting the manuscript and discussing for the findings. All authors have read and approved the final manuscript.

\section{REFERENCES}

Dahlqvist, A., U. Stahl and M. Lenman. 2000. Phospholipid: Diacylglycerol acyltransferase: An enzyme that catalyzes the acyl-CoA-independent formation of triacylglycerol in yeast and plants. Proc. Natl. Acad. Sci. 97: 6487-6492.

Gebauer, S. K., T. L. Psota, W. S. Harris and P. M. Kris-Etherton. 2006. n-3 Fatty acid dietary recommendations and food sources to achieve essentiality and cardiovascular benefits. Am. J. Clin.
Nutr. 83: 1526S-1535S.

Gugel, R. K. and K. C. Falk. 2006. Agronomic and seed quality evaluation of Camelina sativa in western Canada. Can. J. Plant. 86: 1047-1058.

Guy, S. O., D. J. Wysocki, W. F. Schillinger, T. G. Chastain, R. S. Karow, K. Garland-Campbell and I. C. Burke. 2014. Camelina: Adaptation and performance of genotypes. Field Crop Res. 155: 224-232.

Haslam, R. P., O. Sayanova, H. J. Kim, E. B. Cahoon and J. A. Napier. 2016. Synthetic redesign of plant lipid metabolism. Plant J. 87: $76-86$

Iskandarov, U., H. J. Kim and E. B. Cahoon. 2014. Camelina: An emerging oilseed platform for advanced biofuels and bio-based materials. Adv. Plant Biol. 4: 131-140.

Jako, C., A. Kumar, Y. Wei, J. Zou, D. L. Barton, E. M. Giblin, P. S. Covello and D. C. Taylor. 2001. Seed-specific over-expression of an Arabidopsis cDNA encoding a diacylglycerol acyltransferase enhances seed oil content and seed weight. Plant Physiol. 126: 861-874.

Kagale, S., C. Koh, J. Nixon, V. Bollina, W. E. Clarke, R. Tuteja, C. Spillane, S. J. Robinson, M. G. Links and C. Clarke. 2014. The emerging biofuel crop Camelina sativa retains a highly undifferentiated hexaploid genome structure. Nat. Commun. 5: 3706.

Kaup, M. T., C. D. Froese and J. E. Thompson. 2002. A Role for diacylglycerol acyltransferase during leaf senescence. Plant Physiol. 129: 1616-1626.

Kim, H. U., K. R. Lee, Y. S. Go, J. H. Jung, M. C. Suh and J. B. Kim. 2011. Endoplasmic reticulum-located PDAT1-2 from castor bean enhances hydroxy fatty acid accumulation in transgenic plants. Plant Cell Physiol. 52: 983-993.

Li, R., K. Yu and D. F. Hildebrand. 2010. DGAT1, DGAT2 and PDAT expression in seeds and other tissues of epoxy and hydroxy fatty acid accumulating plants. Lipids. 45: 145-157.

Li, R., T. Hatanaka, K. Yu, Y. Wu, H. Fukushige and D. Hildebrand. 2013. Soybean oil biosynthesis: Role of diacylglycerol acyltransferases. Funct. Integr. Genomics. 13: 99-113.

Liu, B. and C. Benning. 2012. Lipid metabolism in microalgae distinguishes itself. Curr. Opin. Biotechnol. 24: 300-309.

Lu, C. and J. Kang. 2008. Generation of transgenic plants of a potential oilseed crop Camelina sativa by agrobacteriummediated transformation. Plant Cell Rep. 27: 273-278.

Lu, C. L., B. D. N. Shen, D. H. Hobbs, J. Kang, Y. Wen, D. Krachtus and M. J. Hills. 2003. Expression pattern of diacylglycerol acyltransferase-1, an enzyme involved in triacylglycerol biosynthesis, in Arabidopsis thaliana. Plant Mol. Biol. 52: 31-41.

Mcvay, K. A. and Q. A. Khan. 2011. Camelina yield response to different plant populations under dryland conditions. Agron. J. 103: 1265-1269.

Petrie, J. R., P. Shrestha, S. Belide, Y. Kennedy, G. Lester, Q. Liu, U. K. Divi, R. J. Mulder, M. P. Mansour and P. D. Nichols. 2014. Metabolic engineering Camelina sativa with fish oil-like levels of DHA. PLoS One. 9: e85061.

Schmittgen, T. D. and K. J. Livak. 2008. Analyzing real-time PCR data by the comparative $C(T)$ method. Nat. Protoc. 3: 1101-1108.

Ståhl, U., A. S. Carlsson and M. Lenman. 2004. Cloning and functional characterization of a phospholipid: Diacylglycerol acyltransferase from Arabidopsis. Plant Physiol. 135: 1324-1335.

Wu, Y., R. Li and D. F. Hildebrand. 2012. Biosynthesis and metabolic engineering of palmitoleate production, an important contributor to human health and sustainable industry. Prog. Lipid. Res. 51: $340-349$. 
Xue, J. A., X. Mao, Z. R. Yang, Y. M. Wu, X. Y. Jia, L. Zhang, A. Q. Yue, J. P. Wang and R. Z. Li. 2013. Expression of yeast acyl-CoA-9 desaturase leads to accumulation of unusual monounsaturated fatty acids in soybean seeds. Biotechnol. Lett. 35: 951-959.

Yang, Y., X. Yu, L. Song and C. An. 2011. ABI4 activates DGAT1 expression in Arabidopsis seedlings during nitrogen deficiency. Plant Physiol. 156: 873-883.

Yuan, L. X., X. Mao and R. Z. Li. 2015. New type of industrial oilseed crop Camelina sativa: From genome to metabolic engineering.
Plant Physiol. J. 51(8): 1204-1216.

Zanetti, F., A. Monti and M. T. Berti. 2013. Challenges and opportunities for new industrial oilseed crops in EU-27: A review. Ind. Crop Prod. 50: 580-595.

Zhang, M., J. Fan, D. C. Taylor and J. B. Ohlrogge. 2009. DGAT1 and PDAT1 acyltransferases have overlapping functions in Arabidopsis triacylglycerol biosynthesis and are essential for normal pollen and seed development. Plant Cell. 21: 3885-3901.

Zubr, J. 1997. Oil-seed crop: Camelina sativa. Ind. Crop Prod. 6: 113-119. 